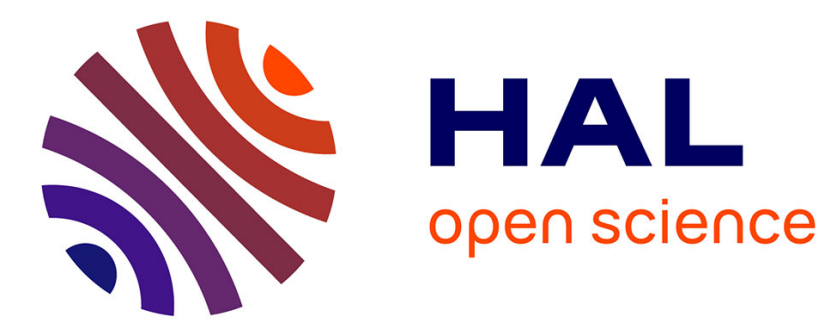

\title{
Experimental and numerical investigations of the aeroacoustics in a corrugated pipe flow
}

Gaëtan Galeron, Daniel Mazzoni, Muriel Amielh, Pierre-Olivier Mattei, Fabien Anselmet

\section{- To cite this version:}

Gaëtan Galeron, Daniel Mazzoni, Muriel Amielh, Pierre-Olivier Mattei, Fabien Anselmet. Experimental and numerical investigations of the aeroacoustics in a corrugated pipe flow. TI 2015 - 4th International Conference on Turbulence and Interactions, Nov 2015, Cargèse, France. hal-01262511

\section{HAL Id: hal-01262511 \\ https://hal.science/hal-01262511}

Submitted on 26 Jan 2016

HAL is a multi-disciplinary open access archive for the deposit and dissemination of scientific research documents, whether they are published or not. The documents may come from teaching and research institutions in France or abroad, or from public or private research centers.
L'archive ouverte pluridisciplinaire HAL, est destinée au dépôt et à la diffusion de documents scientifiques de niveau recherche, publiés ou non, émanant des établissements d'enseignement et de recherche français ou étrangers, des laboratoires publics ou privés. 


\title{
Experimental and numerical investigations of the aeroacoustics in a corrugated pipe flow
}

\author{
Gaëtan GALERON, Daniel MAZZONI, Muriel AMIELH, Pierre Olivier MATTEI, \\ Fabien ANSELMET
}

\begin{abstract}
Our study is focused on the singing risers phenomenon which is encountered in corrugated channels under flow. Internal corrugations are responsible for flow instabilities that synchronize with longitudinal acoustic modes of the channel giving powerful pure tones. Experiments are performed in a specifically designed facility. Numerical simulations of the flow based on a lattice Boltzmann method (LBM) are faced to the experimental results. They aimed at investigating the ability of a LBM based simulation to predict the aeroacoustics of corrugated channels. Acoustic modes and turbulence in the corrugated channel are quite well predicted except the sound pressure levels that need better description of the acoustic boundary conditions.
\end{abstract}

\section{Introduction}

The intense noise generation when passing a flow in a corrugated pipe has intrigued researchers for more than a century [3]. Works carried out in recent years agree to show that the sound generation is related to an interaction between the flow and acoustic resonance in the pipe [10, 12, 4]. The turbulence of the flow, the interaction between the cavities forming the corrugation and finally the feedback whistling of the flow are related to the geometry of the pipe. Due to their flexibility and local stiffness, corrugated pipes are used in many engineering applications. In particular, they are widely used in the oil industry as for the transport of natural gas. Under certain conditions of geometries and flow, the pipes may whistle, generating harmful vibrations to the adjoining industrial facilities. A well-known example is the

Daniel MAZZONI, Muriel AMIELH, Fabien ANSELMET

Aix Marseille Université, CNRS, Centrale Marseille, IRPHE UMR 7342, F-13384, Marseille, e-mail: daniel.mazzoni@centrale-marseille.fr

Gaëtan GALERON, Pierre Olivier MATTEI

CNRS - UPR 7051, F-13453, Marseille, e-mail: gaetan.galeronecentrale-marseille.fr 
"singing riser" phenomenon observed on some natural gas installations in the North Sea.

In our studies, the lengths of the pipes are large with respect to the dimensions of the cavities. The flow at the center of the pipe remains subsonic with velocities corresponding to a Mach number of less than 0.1 . The flow over cavities inducing a sound field is a classic study area [6]. In the case of shallow cavity subjected to an incompressible flow, the hydrodynamic instability of the shear layer that develops on the opening of the cavity is held responsible for the feedback phenomenon [9]. In addition, when, as in our previous study [1], the input of the pipe section has a sufficiently sharp angle, a flow separation occurs near the wall and a veina contracta accelerates the flow on the axis of the pipe. Within this veina contracta, the vortex interaction with a cavity located at a short distance downstream was shown to be the source of strong excitation of pipe longitudinal modes [5].

The aim of the present research is to study both the acoustic and aerodynamic fields within a corrugated pipe under singing conditions. A short description of the especially laboratory designed facility is here given. Simulations with LBM method are faced to experimental results obtained for different flow configurations. Comparisons between experiments and calculations concern velocity and pressure.

\section{Experimental set-up}

For the experimental purpose, a rectangular cross section pipe, $2 \mathrm{~m}$ long, $20 \mathrm{~mm}$ wide, $100 \mathrm{~mm}$ high, has been manufactured (fig 1). It is made by transparent Plexiglas, thereby allowing the use of optical diagnostics like PIV (Particle Image Velocimetry). The 100 wall-cavities are square-shaped of dimensions $10 \times 10 \mathrm{~mm}^{2}$. The upstream edge of each cavity is rounded with the radius $r_{u p}=2.5 \mathrm{~mm}$ while the downstream edge is sharp $\left(r_{\text {down }}=0 \mathrm{~mm}\right)$. These cavities are $10 \mathrm{~mm}$ deep and $10 \mathrm{~mm}$ spaced [1]. Point-wise measurements of velocity and acoustic pressure are made respectively by hot-wire anemometry and by microphone. The velocities measured $10 \mathrm{~mm}$ upstream of the vein entry and downstream, inside the vein, $19 \mathrm{~mm}$ just upstream of the channel exit section, are used for comparisons with the numerical simulation results (fig, 22).

\section{Discrete Boltzmann equation}

The Lattice Boltzmann Method LBM is a recently developed approach used to compute fluid flows. It is based on the kinetic theory for a fluid flow and from the Boltzmann Transport Equation from the classical kinetic theory of gases. The discrete Boltzmann equation with the Bhatnagar-Gross-Krook (BGK) approximation [13] governs the evolution of the probability density function $f_{i}(\mathbf{x}, t)=f\left(\mathbf{x}, \xi_{i}, t\right)$ of finding a particle at the point $x$, at the date $t$, with velocity $\xi_{i}$, in absence of external 


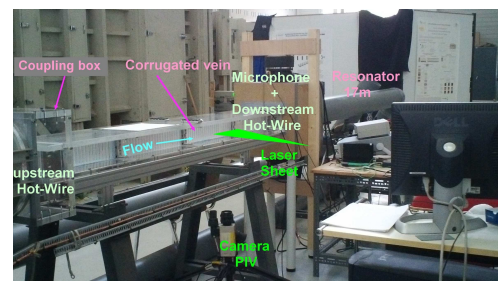

Fig. 1: View of the experiment

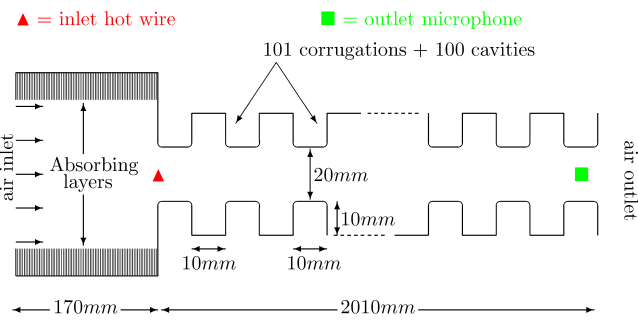

Fig. 2: Computed geometry

forces. For the two dimensional nine velocities lattice (D2Q9) used in the present study, $\xi_{i}$ are the nine possible velocities $(i=0, \ldots, 8)$ for the particles in this discrete velocity space.

$$
\frac{\partial f_{i}}{\partial t}+\xi_{i} \cdot \nabla_{\mathbf{x}} f_{i}=-\frac{1}{\tau}\left[f_{i}-f_{i}^{e q}\right]
$$

where $f_{i}^{e q}$ is the discrete probability density function in absence of any collision, $f_{i}^{e q}$ is usually taken as the second order expansion of the Maxwell velocity distribution leading to:

$$
f_{i}^{e q}(\rho, \mathbf{u})=w_{i} \rho\left(1+\frac{\xi_{i} \cdot \mathbf{u}}{c_{L B}^{2}}+\frac{\left(\xi_{i} \cdot \mathbf{u}\right)^{2}}{2 c_{L B}^{4}}-\frac{\mathbf{u}^{2}}{2 c_{L B}^{2}}\right)
$$

where $\mathbf{u}$ is the local resultant velocity vector. $w_{0}=4 / 9, w_{1, . .4}=1 / 9, w_{5, . ., 9}=1 / 36$ are Gauss integration points. $c_{L B}=1 / \sqrt{3}$ is the lattice constant. $\tau$ is the relaxation time related to fluid viscosity, see eq 6 . The macroscopic fluid density and velocity are the moments of the discrete density function $f_{i}$ :

$$
\begin{aligned}
\text { Mass density: } \rho & =\sum_{i} f_{i}=\sum_{i} f_{i}^{e q} \\
\text { Momentum flux: } \rho \mathbf{u} & =\sum_{i} \xi_{i} f_{i}=\sum_{i} \xi_{i} f_{i}^{e q}
\end{aligned}
$$

Integrating the Boltzmann equation (11) along the characteristics $\xi_{i}$, for a space-step equal to one $(\Delta x=1)$, denoting $\mathbf{e}_{i}$ the unit vector pointing in the direction of the velocity vector $\xi_{i}$, and supposing that the particles move of one cell per time step $\Delta t$ (ie $\Delta x \mathbf{e}_{i}=\Delta t \xi_{i}$ ), one obtains the lattice Boltzmann equation (LBE):

$$
f_{i}\left(\mathbf{x}+\mathbf{e}_{i}, t+\Delta t\right)-f_{i}(\mathbf{x}, t)=-\frac{\Delta t}{\tau}\left[f_{i}(\mathbf{x}, t)-f_{i}^{e q}(\mathbf{x}, t)\right]
$$

Chapman-Enskog analysis [7] shows that in the limit of long-wavelengths, lowfrequency, the LBE reproduces exactly the Navier-Stokes equation for weakly compressible flows with an ideal equation of state that is: $P=\rho c_{s}^{2}$ where $P$ is the pressure and where $c_{s}=343 \mathrm{~ms}^{-1}$ is the speed of sound in air. The kinematic viscosity of the fluid $v$ is:

$$
v=c_{s}^{2}\left(\tau-\frac{\Delta t}{2}\right)
$$


Given the limitation of low Mach number of the LBM, the main interest of the remaining compressibility is to bring the acoustic phenomena. The LBM ability to simulate the propagation of acoustic waves is presented in [2]. Recent developments of the LBM acoustic behaviour are proposed in [15].

\section{Results: comparisons with experiments}

Parallel computations are performed by using the Palabos [11] lattice Boltzmann code with around 50 cores of the mesocentre [8] of Aix Marseille University. The velocity of the particles on the lattice $u_{L B}$ are calculated assuming that the Mach number is conserved so that $u_{L B}=c_{L B} u_{0} / c_{s}$ where $\left(u_{0}, u_{L B}\right)$ and $\left(c_{s}, c_{L B}\right)$ are the flow velocities and the speeds of sound respectively in the physical space and on the lattice. The geometry is a two-dimensional channel $2.01 \mathrm{~m}$ long, $20 \mathrm{~mm}$ wide, with corrugated walls (fig 2). The wall-cavities are similar to the experiment except the upstream and downstream edges with the radii $r_{u p}=5 \mathrm{~mm}$ and $r_{\text {down }}=1 \mathrm{~mm}$. The inlet section of this corrugated channel is connected to a tranquillizer chamber of dimensions $170 \times 100 \mathrm{~mm}^{2}$ with absorbing walls. The role of this tranquillizer chamber is to introduce a sudden change of section of the flow path leading firstly to the flow destabilization and secondly to an approximated Dirichlet acoustic boundary condition. The whole domain is meshed with a $10 \mu \mathrm{m}$ constant step grid. The boundary conditions on the walls are obtained by the "bounce-back" procedure. At the inlet of the domain, a turbulent pipe flow with a $1 / 7$ power law velocity profile is used. At the outlet, a constant density condition is imposed that writes $\rho=1$ in the dimensionless form and insures acoustic Dirichlet boundary conditions.

Seven flow velocity cases are simulated during $1 s$ for $u_{j}=8.4,13,17,21,25$, $29,33 \mathrm{~ms}^{-1}(j=1, . ., 7)$. These reference velocities are those calculated on the axis, $5 \mathrm{~mm}$ downstream of the corrugated channel entry. Acoustic waves effectively travel in the corrugated channel as shown by spectral analysis of the density calculated by the LBM. When the flow velocity increases, successive acoustic modes of the pipe appear (fig 3). The frequencies of the acoustic modes observed on the flow simulations agree with the theoretical eigenfrequencies of an open pipe: $f_{t h_{n}}=n c_{e f f} / 2 L$ with an acceptable relative error lower than 5\%. The efficient speed of sound used for this modal identification, $c_{e f f}=299 \mathrm{~ms}^{-1}$, has been calculated numerically by measuring the velocity of an acoustic plane wave propagating in the corrugated channel and agrees with the experimental value [1]. The turbulence of the velocity field in the numerical simulation is compared at $u_{4}=21 \mathrm{~ms}^{-1}$ to the experiment on fig. 4 At this velocity, a strong whistling was experimentally observed. This comparison is given at the position of the downstream hot-wire (19 $\mathrm{mm}$ upstream of the exit). At this location, the turbulence has developed so that the downstream hot-wire velocity spectrum fits the usual $f^{-5 / 3}$ decay rate. For the numerical simulation, the decay of the turbulence spectrum fits a $f^{-3}$ slope, that is an expected result regarding the two-dimensional geometry [14]. The upstream hot-wire is mainly sensitive to the acoustic velocity. The observed strong peaks correspond to the acoustic eigenfre- 


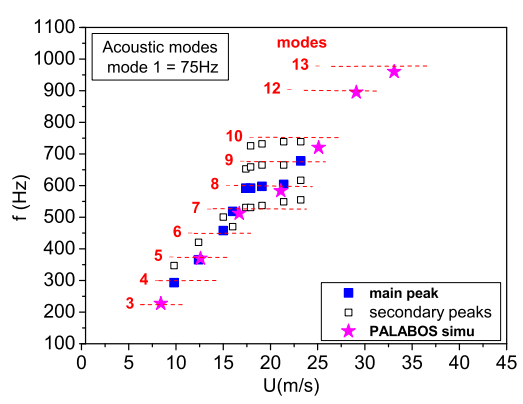

Fig. 3: Acoustic modes in the corrugated pipe

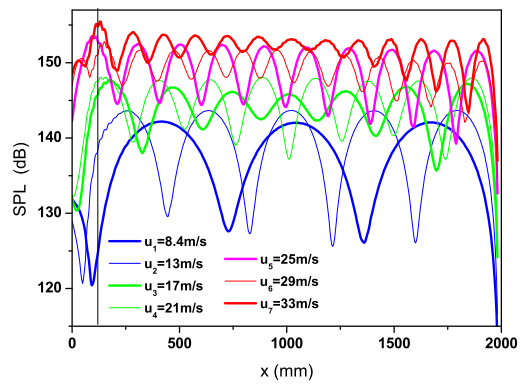

Fig. 5: Simulated acoustic pressure level $(\mathrm{dB})$

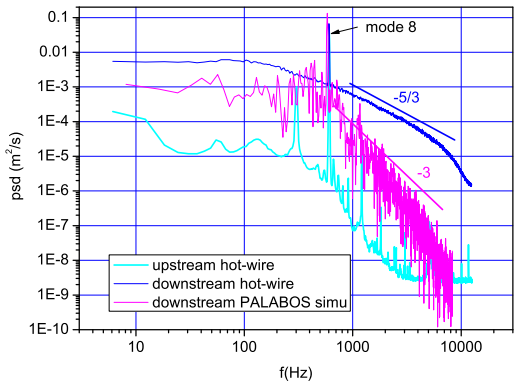

Fig. 4: Spectral analysis

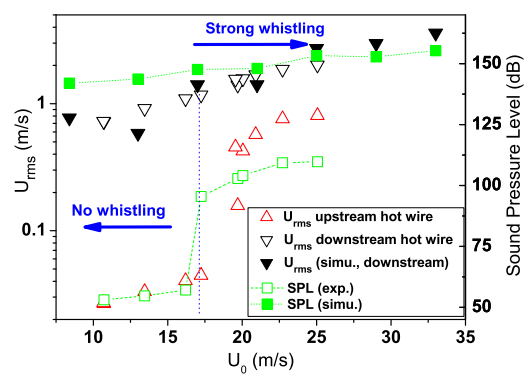

Fig. 6: Comparison of $U_{r m s}$ and SPL with experiment

quencies of the corrugated channel. Corresponding acoustic modes are presented in figure 3. A good agreement between experiment and simulation is obtained with the reference velocities. Figures 5 and 6 concern acoustic (SPL) and turbulence levels $\left(U_{r m s}\right)$. In figure 5 one observes the main acoustic mode for each reference velocity. In the experiment, the whistling occurs from the reference velocity $17 \mathrm{~ms}^{-1}$ with a strong amplification of the SPL and of the $U_{r m s}$ on the upstream hot-wire. This threshold on SPL is not observed on the simulation results, moreover the simulated SPL level is higher than experimental ones. The lack of radiating boundary pressure conditions at the extremities of the pipe induces an overestimation of the internal SPL. Nethertheless, the turbulence velocity levels $U_{r m s}$ are correctly predicted in the downstream part of the corrugated channel. 


\section{Conclusion - Perspectives}

First 2D-computations are performed by using a LBM open source code (Palabos) to predict whistling in a corrugated channel under flow. Results from experiments performed in similar configurations are used for comparisons. Acoustic mode shapes are correctly predicted for the reference velocities. However the acoustic levels are overestimated due to acoustic boundary conditions that have to be improved. Perspectives are now to develop 3D-simulations for better prediction of turbulence and also parametric studies on the influence of corrugation geometry on the whistling.

Acknowledgments - The work presented herein is sponsored by TOTAL and granted access to the HPC resources of Aix Marseille Université financed by the project Equip@ Meso (ANR-10EQPX-29-01) of the program "Investissements d'Avenir" supervised by the ANR.

\section{References}

1. M. Amielh, F. Anselmet, Y. Jiang, U. Kristiansen, P.O. Mattei, D. Mazzoni, C. Pinhède. Aeroacoustic source analysis in a corrugated flow pipe using low-frequency mitigation, J. Turb., 15(10), 650676 (2014).

2. J.M. Buick, C.A. Greated, D.M. Campbell. Lattice BGK simulation of sound waves. Enrophys. Lett., 43(3), 235-240 (1998).

3. W. Burstyn, Eine neue Pfeife (a new pipe). Z. Tech. Phys. Leipzig, 3, 179180 (1922).

4. U.R. Kristiansen, G.A. Wiik. Experiments on sound generation in corrugated pipes with flow, J. Acoust. Soc. Am. 121, 13371444 (2007).

5. U. Kristiansen, D. Mazzoni, A.B. Krogvig. Aeroacoustic investigation of a flow pipe with a small cavity using the lattice Boltzmann method, Proceedings of the 35th Scandinavian Symposium on Physical Acoustics, Geilo, Norway (2012).

6. M.S. Howe. Acoustics of fluid-structure interactions, Cambridge Monographs on Mechanics, Cambridge, University Press (1998)

7. D.O. Martinez, W.H. Matthaeus, S. Chen, D.C. Montgomery. Comparison of spectral method and lattice Boltzmann simulations of two-dimensional hydrodynamics, Phys. Fluids 6, 1285 (1994).

8. https://equipex-mesocentre.univ-amu.fr/

9. S.C. Morris. Shear-Layer Instabilities: Particle Image Velocimetry Measurements and Implications for Acoustics, Annu. Rev. Fluid Mech. 43, 529550 (2011).

10. G. Nakiboglu, H.B.M. Manders, A. Hirschberg. Aeroacoustic power generated by a compact axisymmetric cavity: Prediction of self-sustained oscillation and influence of the depth, J. Fluid. Mech., 703, 163191 (2012).

11. http://www.palabos.org

12. A.M. Petrie, I. D. Huntley. The acoustic output produced by a steady airflow through a corrugated duct, J. Sound Vib. 70, 19 (1980).

13. Y. Qian, D. D'Humieres, P. Lallemand. Lattice BGK Models for Navier-Stokes Equation. Europhys. Lett., 17 (6), 479-484 (1992).

14. P. Tabeling. Two-dimensional turbulence: a physicist approach, Phys. Rep. 362, 1-62 (2002).

15. H. Xu, P. Sagaut. Optimal low-dispersion low-dissipation LBM schemes for computational aeroacoustics, Journal of Comp. Phys. 230, 5353-5382 (2011). 